ICNMM2012-73321

\title{
EVALUATION OF NUSSELT NUMBER FOR A FLOW IN A MICROTUBE WITH SECOND-ORDER MODEL INCLUDING THERMAL CREEP
}

\author{
Barbaros Çetin \\ Mechanical Engineering Department \\ Microfluidics \& Lab-on-a-chip Research Group \\ Bilkent University \\ Ankara 06800 \\ Turkey \\ Email: barbaros.cetin@bilkent.edu.tr, barbaroscetin@gmail.com
}

\begin{abstract}
In this paper, Nusselt number for a flow in a microtube is determined analytically with a constant wall heat flux thermal boundary condition. The flow assumed to be incompressible, laminar, hydrodynamically and thermally fully-developed. The thermo-physical properties of the fluid are assumed to be constant. The effect of rarefaction, viscous dissipation, axial conduction, which are important at the microscale, are included in the analysis. For the implementation of the rarefaction effect, two different second-order slip models are used for the slip-flow and temperature-jump boundary conditions together with the thermal creep at the wall. Closed form solutions for the fully-developed temperature profile and Nusselt number are derived as a function of Knudsen number, Brinkman number and Peclet number.
\end{abstract}

\section{NOMENCLATURE}

$a_{1}$ coefficient defined in Eqn. (1)

$a_{2}$ coefficient defined in Eqn. (1)

$a_{3}$ coefficient defined in Eqn. (1)

$b_{1}$ coefficient defined in Eqn. (2)

$b_{2}$ coefficient defined in Eqn. (2)

$\mathrm{Br}$ Brinkman number

D tube diameter

$\mathrm{k}$ thermal conductivity

Kn Knudsen number
$\mathrm{Nu}$ Nusselt number

$\mathrm{r}$ radial coordinate

$\mathrm{P}$ pressure

Pe Peclet number

Pr Prandtl number

$\dot{q}^{\prime \prime} \quad$ wall heat flux

Re Reynolds number

$\mathrm{R}$ tube radius

$\mathrm{T}$ temperature

u $x$-velocity

$\mathrm{x}$ longitudinal coordinate

$\Gamma$ parameter defined in Eqn. (11)

$\eta$ non-dimensional radial coordinate

$\theta$ non-dimensional temperature

$\kappa$ parameter defined in Eqn. (7)

$\lambda$ mean-free-path

$\mu$ viscosity

$\xi$ non-dimensional longitudinal coordinate

$\Upsilon$ parameter defined in Eqn. (21)

$\phi$ non-dimensional temperature

$\chi$ parameter defined in Eqn. (7)

$\Omega$ parameter defined in Eqn. (13) 


\section{INTRODUCTION}

With the today's fabrication facility, micro-sized fluidic and thermal systems with micrometer dimensions are used in many biomedical and engineering applications such as micro-reactors, micro-heat exchangers, cell reactors etc. For an effective and economical design of such micro-sized systems, the fundamental understanding of the transport phenomena at microscale is crucial. There are several issues that need to be considered at microscale. As the characteristic length $(L)$ of the flow approaches to the mean-free-path $(\lambda)$ of the fluid, the continuum approach fails to be valid, and the fluid flow modeling moves from continuum to molecular model. The ratio of the mean-free-path to the characteristic length of the flow $(L)$ is known as the Knudsen number $(K n=\lambda / L)$. For the $K n$ number varying between 0.01 and 0.1 (which corresponds to the flow of the air at standard atmospheric conditions through the channel that has the characteristic length of $1 \sim 10 \mu \mathrm{m}$ ), the regime is known as the slip-flow regime. In this regime, flow can be modeled with the continuum modeling as far as the boundary conditions are modified to take into account the rarefaction effects.

The general form of the boundary conditions for velocity and temperature can be written as follows:

$$
\begin{gathered}
u-u_{w}=a_{1} \lambda\left(\frac{\partial u}{\partial n}\right)_{w}+a_{2} \lambda^{2}\left(\frac{\partial^{2} u}{\partial n^{2}}\right)_{w}+a_{3} \lambda^{2}\left(\frac{\partial T}{\partial t}\right)_{w} \\
T-T_{w}=b_{1} \lambda\left(\frac{\partial T}{\partial n}\right)_{w}+b_{2} \lambda^{2}\left(\frac{\partial^{2} T}{\partial n^{2}}\right)_{w}
\end{gathered}
$$

where $w$ stand for wall, $n$ and $t$ stand for normal and tangential directions, respectively. First terms of the Eqs. (1) and (2) are known as the first-order boundary conditions, and the second terms are known as the second-order boundary conditions [1]. As the modeling moves to the edge of the slip flow regime (i.e. $K n$ approaches 0.1 ), the inclusion of the second-order terms improves the accuracy of the solution. The last term of the Eqn. (1) is known as the thermal creep. There are two common models for second-order boundary conditions, which were suggested by Karniadakis et al. [1] and Deissler [2]. In this study, these two models are implemented. The corresponding coefficients for these two models are tabulated in Tab. 3 .

The effect of the viscous dissipation, which is characterized by Brinkman number, and the axial conduction, which is characterized by Peclet number, are also important at microscale [3]. The fluid flow [4-7] and heat transfer [3, 8-21] inside a micro-conduit was analyzed for different geometries such as circular tube [3,8-14], parallel plate [12,15-19], rectangular channel [4-7, 20], annular channel [21] using firstorder [3, 6-8, 10-12, 15-17, 19, 21] and second-order models
TABLE 1. LIST OF THE COEFFICIENTS USED IN EQN. (1)

\begin{tabular}{cccc}
\hline & $a_{1}$ & $a_{2}$ & $a_{3}$ \\
\hline Karniadakis et al. [1] & 1.0 & $1 / 2$ & $\frac{3}{2 \pi} \frac{\gamma-1}{\gamma} \frac{c_{p} \rho}{\mu}$ \\
Deissler [2] & 1.0 & $-9 / 8$ & $\frac{3}{2 \pi} \frac{\gamma-1}{\gamma} \frac{c_{p} \rho}{\mu}$ \\
\hline
\end{tabular}

TABLE 2. LIST OF THE COEFFICIENTS USED IN EQN. (2)

\begin{tabular}{ccc}
\hline & $b_{1}$ & $b_{2}$ \\
\hline Karniadakis et al. [1] & $\frac{2-F_{T}}{F_{T}} \frac{2 \gamma}{\gamma+1} \frac{1}{P r}$ & $\frac{2-F_{T}}{F_{T}} \frac{\gamma}{\gamma+1} \frac{1}{P r}$ \\
Deissler [2] & $\frac{2-F_{T}}{F_{T}} \frac{2 \gamma}{\gamma+1} \frac{1}{P r}$ & $-\frac{9}{128} \frac{177 \gamma-145}{\gamma+1}$ \\
\hline
\end{tabular}

$[4,5,13,14,18,20]$. Viscous dissipation $[3,9-12,14,16,17,20]$ and axial conduction $[6,7,11,12,14,16,20]$ are included in some studies .

Thermal creep is the fluid flow in the direction from cold to hot due to the tangential temperature gradient along the channel walls, and observed for rarefied fluids [1]. Thermal creep can enhance or reduce the flowrate in a channel depending on the direction of the tangential temperature gradient at the channel wall. The effect of the thermal creep can be implemented into the model by introducing an additional term in the slip-flow boundary condition as seen in Eqn. (1). The effect of the thermal creep on heat transfer is included in very few studies $[6,7,18-20]$.

In this study, Nusselt number for a flow in a microtube is determined analytically with a constant wall heat flux thermal boundary condition. The flow assumed to be incompressible, laminar, hydrodynamically and thermally fully-developed. The thermo-physical properties of the fluid are assumed to be constant. The effect of rarefaction, viscous dissipation, axial conduction are included in the analysis. For the implementation of the rarefaction effect, two different second-order slip models are used for the slip-flow and temperature-jump boundary conditions together with the thermal creep at the wall. Closed form solutions for the fully-developed temperature profile and Nusselt number are derived as a function of Knudsen number, Brinkman number and Peclet number. 


\section{ANALYSIS}

The steady-state, hydrodynamically-developed flow with a constant temperature, $T_{i}$, flows into the microtube with the constant heat flux at the wall. The non-dimensional governing energy equation including the axial conduction and the viscous dissipation term, and the corresponding boundary conditions can be written as,

$$
\begin{gathered}
\frac{\bar{u}}{2} \frac{\partial \theta}{\partial \xi}=\frac{1}{\eta} \frac{\partial}{\partial \eta}\left(\eta \frac{\partial \theta}{\partial \eta}\right)+\frac{1}{P e^{2}} \frac{\partial^{2} \theta}{\partial \xi^{2}}+2 B r\left(\frac{\partial \bar{u}}{\partial \eta}\right)^{2}, \\
\theta=0 \quad \text { at } \xi=0, \\
\theta \rightarrow \theta_{\infty} \quad \text { as } \xi \rightarrow \infty, \\
\theta \rightarrow \text { finite at } \eta=0, \\
\frac{\partial \theta}{\partial \eta}=1 \quad \text { at } \eta=1,
\end{gathered}
$$

together with the following dimensionless parameters:

$$
\begin{array}{r}
\eta=\frac{r}{R}, \xi=\frac{x}{P e \cdot R}, \theta=\frac{T-T_{i}}{\dot{q}^{\prime \prime} R / k}, \bar{u}=\frac{u}{u_{o}}, \\
u_{o}=\frac{d P}{d x} \frac{R^{2}}{4 \mu}, P e=R e \cdot \operatorname{Pr}, B r=\frac{\mu u_{o}^{2}}{\dot{q}^{\prime \prime} R} .
\end{array}
$$

$\bar{u}$ in Eqn. (3) is the dimensionless fully-developed velocity profile for the slip-flow regime. $\bar{u}$ can be determined by solving the momentum equation together with the slip-velocity boundary condition:

$$
\bar{u}=\frac{u}{u_{o}}=\chi-\eta^{2}+\kappa T_{\xi},
$$

where $\chi$ and $\kappa$ are define as,

$$
\chi=1+4 a_{1} K n-8 a_{2} K n^{2}, \kappa=a_{3} K n^{2} / B r .
$$

The fully-developed temperature profile has the following functional form [1],

$$
\theta_{\infty}=T_{\xi} \xi+\phi(\eta)
$$

where $T_{\xi}$ represents the temperature gradient at the wall $(\partial T / \partial \xi)_{\text {wall }}$ (it is constant for a fully-developed temperature). $T_{\xi}$ can be determined by substituting Eqn. (8) into Eqn. (3), and integrating once in $\eta$-direction together with the boundary condition at the wall as,

$$
T_{\xi}=\frac{1-2 \chi+\sqrt{(1-2 \chi)^{2}+64 \kappa(2 B r+1)}}{4 \kappa} .
$$

Integrating Eqn. (3) in $\eta$-direction together with the boundary condition at the microtube center, $\phi$ can be determined as:

$$
\phi(\eta)=\Gamma r^{2}-\left(B r+\frac{T_{\xi}}{32}\right) \frac{r^{4}}{2}+C,
$$

where $C$ is an arbitrary constant, and $\Gamma$ is defined as,

$$
\Gamma=T_{\xi}\left(\chi+\kappa T_{\xi}\right) .
$$

$C$ can be determined by substituting Eqn. (8) into Eqn. (3) and integrating resulting equation in $\eta$-direction from 0 to 1 , and in $\xi$-direction as,

$$
C=\frac{1}{6}\left(B r+\frac{T_{\xi}}{8}\right)-\frac{\Gamma}{16}+\frac{\Omega}{12}\left(\frac{T_{\xi}}{16}-B r+\frac{48 T_{\xi}}{P e^{2}}\right) .
$$

where $\Omega$ is define as,

$$
\Omega=\frac{1}{2 \chi-1+\kappa T_{\xi}} .
$$

Fully-developed temperature can be obtained by substituting Eqn. (10) into Eqn. (8) as,

$$
\theta_{\infty}(\xi, \eta)=T_{\xi} \xi+\frac{T_{\xi}}{8}\left(\chi+\kappa T_{\xi}\right) \eta^{2}-\left(\frac{B r}{2}+\frac{T_{\xi}}{32}\right) \eta^{4}+C,
$$

where constant $T_{\xi}$ and $C$ are defined in Eqs. (9) and (12), respectively.

Note that to recover the result for the case without thermal creep (i.e. $a_{3} \rightarrow 0$ ), the limit of $T_{\xi}$ needs to be determined. The limit results in,

$$
\lim _{a_{3} \rightarrow 0} T_{\xi}=\frac{8(2 B r+1)}{2 \chi-1}
$$

Macrochannel result (i.e. $K n=B r=0$ ) [22] can be recovered as $^{1}$,

$$
\theta_{\infty}=8 \xi+\eta^{2}-\frac{\eta^{4}}{4}-\frac{7}{24}+\frac{32}{P e^{2}}
$$

\footnotetext{
${ }^{1}$ The coefficients of the $\xi$ term and $1 / P e^{2}$ is slightly differs from that of [22] due to the non-dimensionlization of the velocity with $u_{o}$ instead of $u_{\text {mean }}$.
} 
Fully-developed Nusselt number in terms of nondimensional parameters can be written as,

$$
N u_{\infty} \equiv \frac{h_{\infty} D}{k}=-\frac{2}{\theta_{m}-\theta_{w}},
$$

where $\theta_{m}$ is the non-dimensional mean temperature which is defined as,

$$
\theta_{m}=\frac{2 u_{o}}{u_{m}} \int_{0}^{1} \bar{u} \theta \eta d \eta
$$

and $\theta_{w}$ is the wall temperature, and can be determined by the implementation of the temperature-jump boundary condition, Eqn. (2) as,

$$
\theta_{w}=\theta_{\infty}(\xi, 0)+2 b_{1} K n\left(\frac{\partial \theta}{\partial \eta}\right)_{w}-4 b_{2} K n^{2}\left(\frac{\partial^{2} \theta}{\partial \eta^{2}}\right)_{w} .
$$

Fully-developed Nusselt number can be determined as,

$$
\begin{aligned}
N u_{\infty}=-2 /\{ & \frac{1}{2}\left(B r+\frac{T_{\xi}}{16}\right)-\frac{\Gamma}{8}+\Omega \Upsilon-2 b_{1} K n \\
& \left.-4 b_{2} K n^{2}\left(6 B r+\frac{3 T_{\xi}}{8}-\frac{\Gamma}{4}\right)\right\},
\end{aligned}
$$

where $\Upsilon$ is defined as,

$$
\Upsilon=\frac{T_{\xi}}{8}\left(\chi^{2}+\chi T_{\xi} \kappa+\kappa \Gamma+\frac{1}{8}\right)-\frac{5 \Gamma}{48}+B r\left(\frac{1}{4}-\frac{\Gamma}{3 T_{\xi}}\right)
$$

For a macrochannel flow (i. e. $K n=B r=\kappa=0, \chi=1$ ), the solution recovers well-known result of 48/11 [23].

\section{RESULTS AND DISCUSSION}

The fully-developed temperature profile and the fullydeveloped $N u$ is determined. Second-order boundary conditions are implemented to include the rarefaction effects and thermal creep. The viscous dissipation and the axial conduction are also included. Coefficient $b_{1}$ is taken as 1.667 , and $\gamma$ is taken as 1.4 in the calculation of coefficient $b_{2}$, which are typical values for air being the working fluid in many engineering problems.

Fully-developed $\mathrm{Nu}$ is functions of $\mathrm{Kn}, \mathrm{Br}$ and thermal creep as seen from Eqn. (20). It is not function of $P e$, which means $P e$ number only effects the local $N u$ in the thermal entrance region. The fully-developed $\mathrm{Nu}$ for different $\mathrm{Kn}$ and $\mathrm{Br}$ numbers is also

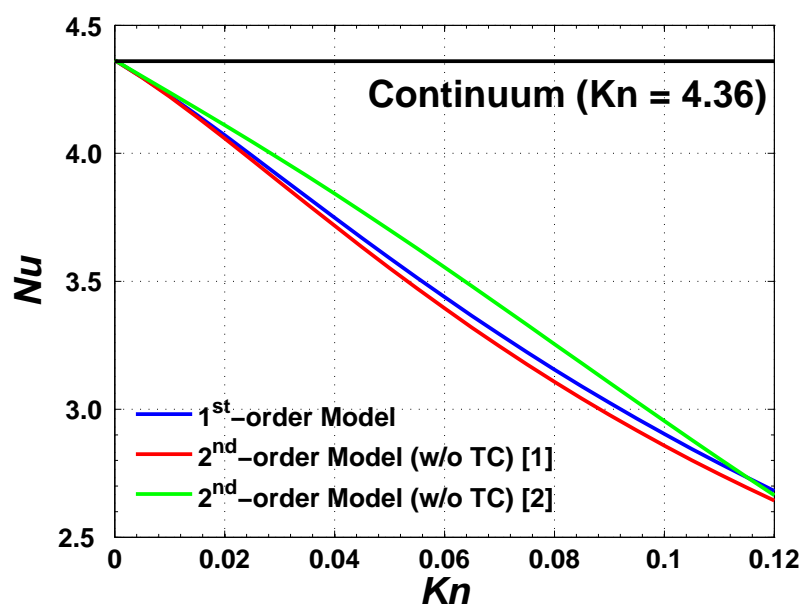

(a) $\mathrm{Br}=0$

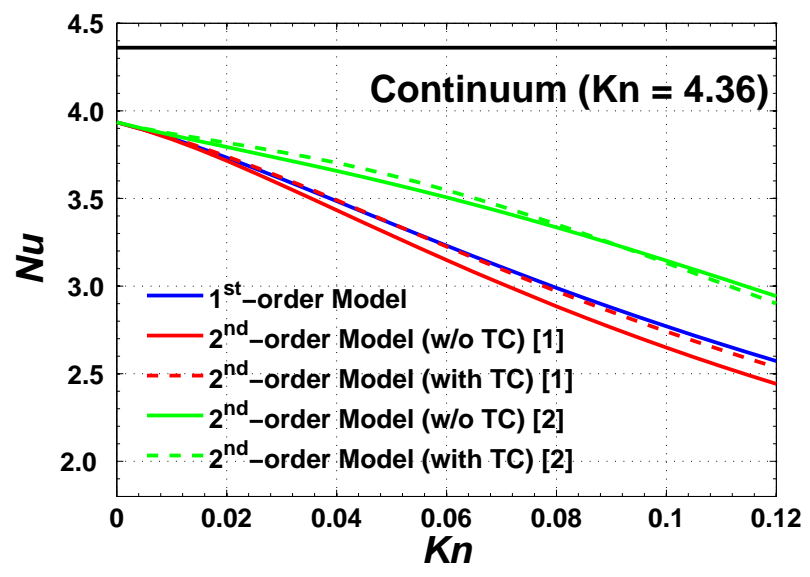

(b) $B r=0.1$

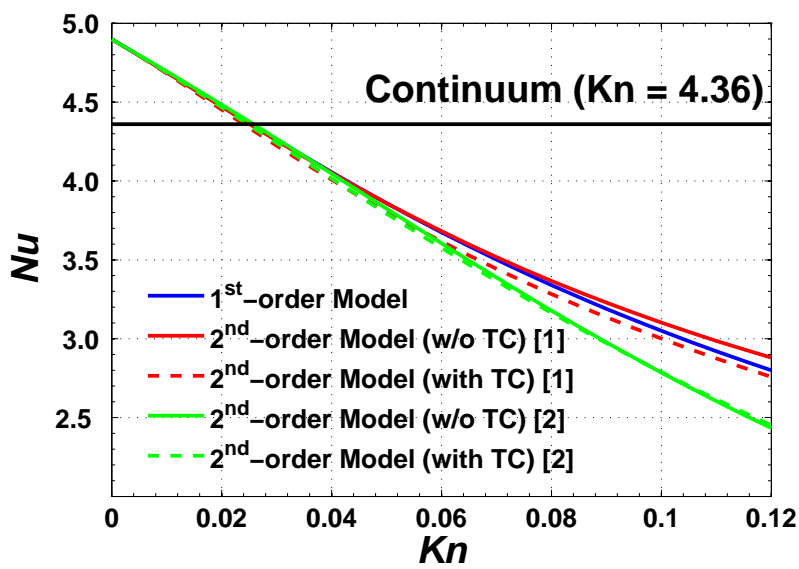

(c) $\mathrm{Br}=-0.1$

FIGURE 1. Variation of the fully-developed $\mathrm{Nu}$ as a function of $K n$ for different $B r$ with and without thermal creep effect (a) $B r=0$, (b) $B r=0.1$, (c) $B r=-0.1$ 
shown in Fig. (1). The results for first-order model are also included in the figures.

For the case of $B r=0$, Fig. 1-(a), the second-order model proposed by Karniadakis et al. [1] gives close results to firstorder model. The deviation from the first-order model increases with increasing rarefaction (i.e. increasing $\mathrm{Kn}$ ). On the other hand, the second-order model proposed by Deissler [2] gives appreciably deviation from first-order model. The deviation increases first, but decreases as $\mathrm{Kn}$ reaches the edge of the slip-flow regime. For the case of $B r=0$ the model with the inclusion of the thermal creep is not included in the figure, since $B r=0$ gives infinite thermal creep. Physical explanation is that if there exist a rarefaction effect, there also exists a viscous dissipation up to certain extent. Although in these figures, $B r$ and $K n$ varying independent of each other, in many engineering applications this is not the case. In the engineering application with microchannels, the devices typically operates in the vicinity of the atmospheric conditions, which means increase in the $K n$ indicates the reduction in the size of the channel. $B r$ has also size dependence.

$\mathrm{Br}$ number has an appreciable effect on $N u$ value. Positive $\mathrm{Br}$ means that the fluid is being heated, and negative $\mathrm{Br}$ means that the fluid is being cooled. Figures 1-(b) and 1-(c) illustrates the cases for $B r=0.1$ and $B r=-0.1$, respectively. In these cases, both cases with and without thermal creep are included in the figures. Likewise Fig. 1-(a), the deviation of the second-order model proposed by Deissler [2] is higher that of the second-order model proposed by Karniadakis et al. [1]. With the inclusion of the thermal creep, fully-developed $N u$ obtained by the secondorder model proposed by Karniadakis et al. [1] approaches the results of the first-order slip model. However, for the secondorder model proposed by Deissler [2], fully-developed $\mathrm{Nu}$ obtained with the inclusion of the thermal creep is very close the results of the cases without thermal creep.

Present analysis has some limitations. The thermo-physical properties of the fluid is assumed to be constant which means the variation of the temperature in the channel should not exceed certain limits. The flow is modeled as incompressible. This is very restrictive, and actually incompressible approach is theoretically inconsistent to model slip-boundary conditions [1]. Therefore, the results of this study should be regarded qualitative rather than quantitative. However, this kind of analytical solutions are useful to reveal the fundamental aspects of the convective heat transfer mechanism.

\section{SUMMARY}

In this study, Nusselt number for a flow in a microtube is determined analytically with a constant wall heat flux thermal boundary condition. The flow assumed to be incompressible, laminar, hydrodynamically and thermally fully-developed. The thermo-physical properties of the fluid are assumed to be constant. The effect of rarefaction, viscous dissipation, axial con-
TABLE 3. FULLY-DEVELOPED NU VALUES

\begin{tabular}{|c|c|c|c|c|c|c|}
\hline \multirow{2}{*}{ Kn } & \multirow{2}{*}{$\begin{array}{c}1^{s t} \text {-order } \\
\text { Model }\end{array}$} & \multicolumn{2}{|c|}{$2^{\text {nd }}$-order Model [1] } & \multicolumn{2}{|c|}{$2^{\text {nd }}-$ order Model [2] } & \multirow{2}{*}{$\mathbf{B r}$} \\
\hline & & w/o TC & with $T C$ & w/o TC & with $T C$ & \\
\hline $\mathbf{0 . 0}$ & 3.934 & 3.934 & 3.934 & 3.934 & 3.934 & \multirow{7}{*}{0.1} \\
\hline 0.02 & 3.733 & 3.715 & 3.741 & 3.793 & 3.818 & \\
\hline 0.04 & 3.485 & 3.433 & 3.490 & 3.657 & 3.507 & \\
\hline 0.06 & 3.231 & 3.149 & 3.225 & 3.507 & 3.548 & \\
\hline 0.08 & 2.990 & 2.885 & 2.972 & 3.335 & 3.353 & \\
\hline 0.10 & 2.770 & 2.649 & 2.741 & 3.145 & 3.132 & \\
\hline 0.12 & 2.572 & 2.442 & 2.536 & 2.942 & 2.901 & \\
\hline $\mathbf{0 . 0}$ & 4.898 & 4.898 & 4.898 & 4.898 & 4.898 & \multirow{7}{*}{-0.1} \\
\hline 0.02 & 4.475 & 4.471 & 4.452 & 4.484 & 4.467 & \\
\hline 0.04 & 4.056 & 4.052 & 4.001 & 4.047 & 4.015 & \\
\hline 0.06 & 3.674 & 3.682 & 3.616 & 3.605 & 3.567 & \\
\hline 0.08 & 3.340 & 3.367 & 3.283 & 3.178 & 3.164 & \\
\hline 0.10 & 3.050 & 3.102 & 3.001 & 2.785 & 2.789 & \\
\hline 0.12 & 2.780 & 2.880 & 2.758 & 2.434 & 2.452 & \\
\hline
\end{tabular}

duction are included in the analysis. For the implementation of the rarefaction effect, two different second-order slip models are used for the slip-flow and temperature-jump boundary conditions together with the thermal creep at the wall. Closed form solutions for Nusselt number are derived as a function of $\mathrm{Kn}$ and $\mathrm{Br}$ number. The results reveal that thermal creep has significant effect on the heat transfer characteristics, and the effect of thermal creep differs for two models. The limitations of the current model are also discussed.

\section{REFERENCES}

[1] Karniadakis, G. E., Beskok, A., and Aluru, N., 2005. Microflows and Nanoflows: Fundamentals and Simulations. Springer, pp. 51-74,167-172.

[2] Deissler, R. G., 1964. "An analysis of second-order slip fow and temperature-jump boundary conditions for rarefied gases". Int. J. Heat Mass Transfer, 7, pp. 681-694.

[3] Cetin, B., Yazicioglu, A., and Kakac, S., 2008. "Fluid flow in microtubes with axial conduction including rarefaction and viscous dissipation". Int. Comm. Heat and Mass Transfer, 35, pp. 535-544.

[4] Aubert, C., and Colin, S., 2001. "High-order boundary conditions for gaseous flows in rectangular microducts". Microscale Thermophysical Eng., 5, pp. 41-54.

[5] Colin, S., 2004. "Validation of a second-order slip flow model in rectangular microchannels". Heat Transfer Eng., 25(3), pp. 23-30. 
[6] Niazmand, H., Amiri-Jaghargh, A., and Renksizbulut, M., 2010. "Slip-flow and heat transfer in isoflux rectangular microchannels with thermal creep effects". J. Appl. Fluid Mech., 3(2), pp. 33-41.

[7] Amiri-Jaghargh, A., Niazmand, H., and Renksizbulut, M., 2010. "Cooling in a constant wall tempertaure microchannels with thermal creep effects". In Proceedings of the ASME $20108^{\text {th }}$ International Conference on Nanochannles, Microchannels and Minichannels, ICNMM2010, August 1-5, 2010, Montreal, Canada, no. 30770 .

[8] Ameel, T. A., Barron, R. F., Wang, X. M., and Warrington, R. O., 1997. "Laminar forced convection in a circular tube with constant heat flux and slip flow". Microscale Thermophysical Eng., 1, pp. 303-320.

[9] Chen, C. S., and Kuo, W. J., 2004. "Heat transfer characteristics of gaseous flow in long mini- and microtubes". Numerical Heat Transfer Part A, 46(5), pp. 497-514.

[10] Aydin, O., and Avc1, M., 2006. "Analysis of micro-Graetz problem in a microtube". Nanoscale and Microscale Thermophysical Engineering, 10(4), pp. 345-358.

[11] Cetin, B., Yazicioglu, A., and Kakac, S., 2009. "Slipflow heat transfer in microtubes with axial conduction and viscous dissipation-An extended Graetz problem". Int. J. Thermal Sciences, 48, pp. 1673-1678.

[12] Çetin, B., Yuncu, H., and Kakac, S., 2006. "Gaseous flow in microchannels with viscous dissipation". Int. J. Transport Phenomena, 8, pp. 297-315.

[13] Xiao, N., Elsnab, J., and Ameel, T., 2009. "Microtube gas flows with second-order slip flow and temperature jump boundary conditions". Int. J. Thermal Sciences, 48(2), pp. 243-251.

[14] Cetin, B., and Bayer, O., 2011. "Evaluation of Nusselt number for a flow in a microtube using second-order slip model". Thermal Sciences, 15 Suppl. 1, pp. 103-109.

[15] Xue, H., Ji, H., and Shu, C., 2003. "Prediction of flow and heat transfer characteristics in micro-Couette flow". $M i$ croscale Thermophysical Eng., 7(1), pp. 51-68.

[16] Jeong, H. E., and Jeong, J. T., 2006. "Extended Graetz problem including streamwise conduction and viscous dissipation in microchannels". Int. J. Heat Mass Transfer, 49, pp. 2151-2157.

[17] Roy, S., and Chakraborty, S., 2007. "Near-wall effects in micro scale Couette flow and heat transfer in the Maxwellslip regime". Microfluid Nanofluid, 3(4), pp. 437-449.

[18] Niazmand, H., and Rahimi, B., 2010. "High order slip and thermal creep effects in micro channel natural convection". In Proceedings of the ASME $20108^{\text {th }}$ International Conference on Nanochannles, Microchannels and Minichannels, ICNMM2010, August 1-5, 2010, Montreal, Canada, no. 30688.

[19] Weng, H. C., and Chen, C.-K., 2008. "On the importance of thermal creep in natural convection gas microflow with wall heat fluxes". J. Phys. D: Appl. Phys., 41, p. 115501.

[20] van Rij, J., Ameel, T., and Harman, T., 2009. "An evaluation of secondary effects on microchannel frictional and convective heat transfer characteristics". Int. J. Heat and Mass Transfer, 52, pp. 2792-2801.

[21] Duan, Z., and Muzychka, Y. S., 2008. "Slip flow heat transfer in annular microchannels with constant heat flux". $J$. Heat Transfer, 130, p. 092401.

[22] Vick, B., and Ozisik, M. N., 1981. "An exact analysis of low Peclet number heat transfer in laminar flow with axial conduction". Letters in Heat and Mass Transfer, 8, pp. 110.

[23] Deen, W. M., 1998. Analysis of Transport Phenomena. Oxford University Press, pp. 391-392. 